\title{
IL N'y Avait Pas D'autre Choix Possible : Conformisme, CONFORMite Et Confirmation - Une APPROChe Psychanalytique Du DeVENIR-Desistant Ou Du DeVEnIR-PERSistant.
}

Jean-Michel Vives1

\section{RÉSUMÉ}

À partir d'une différenciation précise du sens des termes de conformisme, conformité et confirmation, l'auteur s'attache à décrire les mécanismes psychiques en jeu dans le devenir-désistant (ce qui implique de céder sur son désir) et dans le devenir-persistant (qui aurait à voir avec la perlaboration du désir). Ce parcours du conformisme à la confirmation, permet de préciser l'aphorisme lacanien concernant l'éthique de la psychanalyse : "As-tu agi en conformité avec ton désir ?"

MotS-CLÉs: conformisme, conformité, confirmation, désir, éthique, surmoi, voix. 
“II N'y Avait Pas D'autre Choix Possible » : Conformisme, Conformite Et confirmation: Une Approche Psychanalytique Du Devenir-Desistant Ou Du Devenir-Persistant.

Mon intervention trouve son origine dans la surprise, pour ne pas dire la sidération, qui fut la mienne lorsque j'ai entendu une collègue psychanalyste brésilienne affirmer, à l'occasion d'une prise de parole depuis le public, au cours de la journée organisée en hommage à Alain Didier-Weill par l'association Insistance au New Morning, à Paris le 9 juin 2019, qu'elle avait voté pour Jair Messias Bolsonaro et qu'elle justifia ce vote par un argument sans appel : " II n'y avait pas d'autre choix possible ». Sa prise de position dans cette assemblée ne manquait pas de courage. Un murmure se fit entendre parmi les personnes présentes mais celles-ci furent assez délicates pour ne pas insister. Mon propos n'est pas ici de juger le choix de cette collègue: ne vivant pas au Brésil, il m'est difficile d'évaluer ce que peut être le quotidien de ses habitants qui a pu conduire un nombre suffisant de brésiliens à porter jusqu'à la fonction présidentielle un personnage qui, vu depuis la France, relève plus du clown que du Messie.

Or, comme les patients souffrant de coulrophobie - c'est à dire de phobie des clowns, comme une grande majorité d'entre vous j'imagine actuellement peut en souffrir - l'ont très justement repéré - sans avoir jamais vu le film Ça2: derrière la figure du clown peut se révéler une incarnation du surmoi féroce et obscène. Le clown, qui peut à l'occasion prendre l'aspect d'un personnage providentiel, est alors, inconsciemment, le lieu de cette instance sans réel pouvoir et pourtant terrifiante qu'est le surmoi et qui ne cesse de nous proposer la voie de la désistance.

C'est pour éclairer cette dimension que je m'attacherai à l'énigmatique et abrupte justification apportée par cette collègue : « II n'y avait pas d'autre choix possible ». Mon trouble ne venait pas seulement du choix politique de cette collègue. En effet, ayant de nombreux «amis » brésiliens sur FaceBook, j'avais déjà pu constater qu'un nombre étonnamment élevé à mes yeux de psychanalystes brésiliens pouvait soutenir activement cette candidature disons... atypique. Dans un premier temps, j'avais caressé l'espoir qu'il ne s'agissait là que de quelques collègues de l'IPA égarés. Que mes collègues de cette vénérable institution veuillent bien me pardonner cette pensée qui révèle plus mes préjugés que la réalité... De fait, il n'en était rien et je repérai rapidement que des collègues analystes se réclamant de l'enseignement de King, Ça qui met en scène un clown maléfique et tueur. 
Lacan pouvaient également soutenir cette candidature ou au moins s'y rallier « car il n'y avait pas d'autre choix possible ». Voilà un premier paradoxe passionnant : il serait donc possible de lire Lacan, de soutenir avec lui l'existence d'un choix éthique dans le cadre de la cure et parallèlement de déclarer, en dehors du cabinet, qu'il n'y a pas d'autre choix possible.

Qu'est-ce qui conduit un psychanalyste qui s'est découvert au cours de son parcours analytique fille ou fils de la parole et dont la pratique quotidienne est sous tendue par la supposition que justement un autre choix que celui du symptôme, de l'inhibition ou de l'angoisse est possible à affirmer en dehors de son cabinet : « II n'y avait pas d'autre choix possible »?

Je m'intéresserai ici à essayer de déterminer ce qui conduit un sujet à choisir de s'engager dans ce que l'on pourrait nommer un devenir-désistant ou un devenirpersistant. Pour cela, je propose de différencier trois notions qui pourraient, à première vue, paraître proches mais qui impliquent néanmoins des positions subjectives bien différentes et qui sous-tendent le trajet de la désistance à la persistance. Ces trois notions sont le conformisme, la conformité et la confirmation.

L'hypothèse que je vous soumets aujourd'hui est la suivante : l'impossibilité de soutenir qu'un autre choix est possible serait la conséquence d'un mouvement de désistement subjectif, expression de la soumission à l'injonction : « Sois conforme ! », qui apparaitrait lorsque la confirmation du choix d'agir en conformité avec son désir est impossible. J'insiste encore une fois, il ne s'agit pas ici de juger le vote de cette collègue mais d'essayer de comprendre la position subjective conformiste qui le soustend et qui - entendez bien, cela est essentiel pour suivre ce que je vais tenter de vous dire aujourd'hui - nous concerne tous. II y a un potentiel 3 admirateur en attente du Messie en chacun d'entre nous et il est important de le repérer pour justement ne pas s'y réduire. Pour reprendre une formulation chère à Alain Didier-Weill, nous pourrions dire : Je ne suis pas que cette attente infantile d'une figure messianique qui viendrait me décharger d'avoir à désirer et donc de me confronter à la castration. Encore une fois, entendez bien : à partir du double mouvement de négation, porté par le « ne » et le «pas que », le sujet qui soutient cet énoncé reconnaît que cette dimension existe

3 Dans son ouvrage Aurais-je été résistant ou bourreau ? Pierre Bayard interroge de façon très intéressante l'existence d'une personnalité potentielle qui pourrait se révéler dans des conditions extrêmes. Bayard P. (2013) Aurais-je été résistant ou bourreau ? Paris, Éditions de minuit. p. 21-27. 
“II N'y Avait Pas D'autre Choix Possible » : Conformisme, Conformite Et confirmation: Une Approche Psychanalytique Du Devenir-Desistant Ou Du Devenir-Persistant.

bien en lui - ce qui déchoit du symbolique, n'y est pas encore échu ou ne sera jamais traité par lui - mais qu'il est possible de ne pas s'y réduire même si cela n'est pas toujours aisé. Car, comme le rappelle Lacan, à l'occasion du Séminaire Livre VII, le choix d'une éthique héroïque n'est pas sans risque et peut-être, à partir de là, ce choix éminemment subjectif et donc solitaire, n'est-il possible ni pour tous, ni même à tous les moments de notre existence.

Ainsi Lacan nous avertit-il :

L'accès au désir nécessite de franchir non seulement toute crainte, mais toute pitié (...). On sait ce qu'il en coûte de s'avancer dans une certaine direction, et mon Dieu, si on n'y va pas, on sait pourquoi. On peut même pressentir que si on n'est pas tout à fait au clair de ses comptes avec son désir, c'est parce qu'on n'a pas pu mieux faire. Car ce n'est pas une voie où l'on puisse s'avancer sans rien payer (...). Pour celui qui s'avance à l'extrême de son désir, tout n'est pas rose (LACAN, 1959-1960/1997, P. 378).

Puis il ajoute que, pour autant, le sujet en désistance n'est pas dupe de «la valeur de la prudence qui s'oppose (à cet engagement sur la difficile voie du désir), sur la valeur toute relative des raisons bénéfiques, des attachements, des intérêts pathologiques (...) qui peuvent le retenir sur cette voie risquée »4. Pour le dire autrement, la voie du désir n'est pas forcément une partie de plaisir et on peut comprendre que l'on puisse hésiter à s'y engager car, comme le rappelle Lacan, « il est plus commode de subir l'interdit que d'encourir la castration $\gg 5$. C'est ce mouvement de désistement conduisant à préférer l'interdit à la castration que je propose de nommer conformisme.

\section{Du CONFORMISME}

L'étymologie du mot conformisme nous renvoie à l'idée de semblable : con(cum, avec) et forme. Être conforme c'est se soumettre et se glisser dans une forme imposée depuis l'extérieur. L'une des plus saisissantes illustration du conformisme est la célèbre série d'expériences en psychologie sociale conduite entre 1928 et 1950 par Stanley Milgram, professeur de psychologie à l'Université de New-York. Son but était de montrer les conditions de la soumission à une autorité. Feignant de mener une recherche sur la mémoire, Stanley Milgram demande à des personnes d'infliger des chocs électriques d'une intensité croissante à un sujet supposé testé et qui est en fait 
un comparse-comédien. Celui-ci, soit disant objet de l'expérience, est sanglé sur une chaise, une électrode fixée au bras. II doit restituer des listes de couples de mots apprises. Chaque erreur, prévue dans le dispositif expérimental, est sanctionnée par une décharge électrique d'intensité croissante. Les fausses décharges électriques seraient pratiquées par l'intermédiaire d'une machine comportant trente manettes échelonnées de quinze à quatre cent cinquante volts, avec des mentions allant de « choc léger » à « attention : choc dangereux »; les comparses mimant la peur et la douleur consécutives aux décharges électriques factices administrées. Sous couvert de tester des procédés de mémorisation, l'expérience éprouvait, en fait, la soumission à un protocole qui ne faisait que mettre en scène des séances de torture. Or, la grande majorité des personnes accepta de mener l'expérience jusqu'au bout, c'est à dire d'infliger des chocs potentiellement mortels. Ces résultats troublants conduisirent Stanley Milgram à conclure que « des gens ordinaires, dépourvus de toute hostilité, peuvent, en s'acquittant simplement de leur tâche, devenir les agents d'un atroce processus de destruction $» 6$. Puis il ajoute :

\footnotetext{
Ce qui se révèle surprenant, c'est de constater jusqu'où peut aller la soumission d'un individu ordinaire aux injonctions de l'expérimentateur. À vrai dire, les résultats de l'expérience sont à la fois inattendus et inquiétants. Même si l'on tient compte du fait que beaucoup de sujets éprouvent un stress considérable et que certains protestent auprès de l'expérimentateur, il n'en demeure pas moins qu'une proportion importante d'entre eux continue jusqu'au niveau de choc le plus élevé du stimulateur (MILGRAM, 1974 [2017] p. 38).
}

On ne peut que penser ici à ce que Hannah Arendt a pu qualifier de «banalité du mal » dans son ouvrage Eichmann à Jérusalem7.

Ce qui est tout à remarquable ici est le fait que le conformisme implique, chez un public que rien à priori ne destinait à occuper une place de bourreau, l'abandon de toute liberté de penser pour s'en remettre à la volonté d'un autre avec laquelle pourtant on peut être profondément en désaccord. Ce second paradoxe est digne d'intérêt : le conformiste n'est pas forcément d'accord avec l'énoncé qu'il profère ou auquel il se soumet. II se révèle alors porte-parole au sens fort du terme. La parole qu'il soutient peut être en désaccord avec ses principes moraux ou éthiques, il la supporte dans tous les sens du terme car il lui semble qu'une autre voie/voix n'est pas possible. 
“II N'y Avait Pas D'autre Choix Possible » : Conformisme, Conformite Et confirmation: Une Approche Psychanalytique Du Devenir-Desistant Ou Du Devenir-Persistant.

Cela ne saurait, malheureusement pas, étonner les psychanalystes et déjà, dans une lettre du 4 janvier 1928 adressée à Ferenczi, Freud déplorait cette tentation du conformisme qu'il pouvait repérer jusque chez ses élèves.

Les conseils sur la technique que j'ai écrits il y a longtemps ont essentiellement un effet négatif. J'ai considéré qu'il fallait avant tout souligner ce que l'on ne devait pas faire et mettre en évidence les tentations capables de contrarier l'analyse. (...) II en résulta que les analystes dociles ne saisirent pas l'élasticité des règles que j'avais formulées et qu'ils y obéissent comme si elles étaient tabous( FREUD, cité par JONES, 1955/2006, p. 256.)

Quelques mois plus tard, dans une lettre du 25 novembre 1928 à son ami le pasteur Pfister, Freud affirme avec force qu'il veut protéger l'analyse contre les médecins et contre les prêtress, souhaitant par là protéger la psychanalyse de deux types de conformisme qui pourraient se mettre en travers du processus analytique : la furor sanandi médicale d'une part et la moralisation normalisante religieuse d'autre part.

Ce que Freud découvre avec stupéfaction et un peu d'amertume c'est que l'expérience de l'analyse ne préserve pas forcément de la dérive conformiste. Cela n'a rien de rassurant mais il convient de regarder le phénomène sans se détourner pour essayer de comprendre ce qu'il révèle de l'humaine condition et d'une des limites de la psychanalyse.

Pour faire un pas supplémentaire intéressons-nous à cette figure mythique du conformisme qu'est Ismène, la sœur d'Antigone, qui à l'occasion de la première scène de l'œuvre de Sophocle répond les mots suivants à sa sœur qui lui demande de l'aider à enterrer leur frère Polynice à qui Créon refuse une sépulture.

ISMÈNE : «Et, aujourd'hui encore, où nous restons toutes deux seules, imagine la mort misérable entre toute dont nous allons périr, si rebelles à la loi, nous passons outre à la sentence, au pouvoir absolu d'un roi. Rends-toi compte d'abord que nous ne sommes que des femmes : la nature ne nous a pas faites pour lutter contre les hommes; ensuite que nous sommes soumises à des maîtres, et dès lors contraintes d'observer leurs ordres - et cela et de plus durs encore... Pour moi, en tout cas, je supplie les morts sous la terre de m'être indulgents, puisqu'en fait je cède à la force ; mais j'entends obéir au pouvoir établi »( SÓFOCLES, 1973, p. 95).

8 Freud S, (1909-1939) Correspondance avec le pasteur Pfister, lettre du 25/11/1928. Paris, Gallimard. 1966, p. 183. 
Les arguments avancés par Ismène pour tenter de convaincre sa sœur Antigone de ne pas enterrer leur frère pourraient aisément se résumer à un : « II nous faut renoncer pour rester en vie : il n'y a pas d'autre choix possible ». L'intérêt du positionnement d'Ismène est qu'il met en évidence ce que Christopher Browning a proposé de qualifier de conformisme de groupe à partir de l'analyse qu'il fait du comportement d'un bataillon de la police allemande à qui il fut demandé, le 11 juillet 1942, d'assassiner sur place, femmes, enfants et vieillards, après une rafle en Pologne de 1800 juifs. Ce conformisme de groupe aurait selon Browning un rôle important dans la transformation de ces Hommes ordinaires - c'est le nom de son ouvrage - en assassins. Je le cite: «Rompre les rangs 9, faire un pas en avant, adopter un comportement non conformiste était tout simplement au-dessus de leurs forces. Ils trouvaient plus facile de tirer »10. Pour eux également, il semblerait qu' « il n'y avait pas d'autre choix possible ». Dans ce cas comme dans celui d'Ismène, nous pourrions qualifier ce conformisme de conformisme de vie11 qui implique que face à la castration radicale que représente la mort le moi fait le choix du narcissisme pour se maintenir en vie.

Qu'est-ce qui conduit l'homme à se soumettre à ce // impersonnel qui nous décharge d'avoir à choisir? Cette soumission, au-delà du tyran politique, est soumission à un autre despote bien plus pernicieux car, instance intrapsychique que nous partageons tous, il peut se révéler à l'occasion un très efficace allié du tyran politique. Vous aurez reconnu ici la figure du surmoi dans ses dimensions « obscène et féroce»12 mises en évidence par Mélanie Klein et développées par Lacan. « II n'y avait pas d'autre choix possible», est l'expression d'un désistement qui révèle comment un sujet fut-il analysé, voire analyste, peut se désengager de sa responsabilité non seulement envers les devoirs inhérents à l'exercice de la parole mais également envers ce que son analyse lui a permis d'expérimenter :

- premièrement, qu'il n'y a de monde que gagné sur l'immonde. Immonde qu'il convient d'entendre ici dans son sens étymologique qui bien que non avéré est

9 On sait que Antigone face à Ismène et au chœur qui lui demande de se conformer « rompra les rangs ».

10 Browning C., (1992) Des hommes ordinaires. Paris, Tallandier / Texto. 2007, p. 270.

11 Vinot F., Vives J.-M., (2016) «Conformisme de vie, conformisme de mort », Topiques, Conformisme et conservatisme, $\mathrm{n}^{\circ}$ 136. p. 91-101.

12 Lacan J., (1955) « La chose freudienne ou Sens du retour à Freud en psychanalyse », Écrits. Paris, Seuil. 1966, p. 434.

Psicanálise \& Barroco em revista | v.17, n. 3 | dezembro de 2019 
“II N'y Avait Pas D'autre Choix Possible » : Conformisme, Conformite Et confirmation: Une Approche Psychanalytique Du Devenir-Desistant Ou Du Devenir-Persistant.

éclairant: un monde négativé par le préfixe -im. L'im-monde serait ce qui n'aurait pas encore accédé à la dimension de monde habitable. L'immonde serait alors à entendre comme un des noms du réel.

- deuxièmement, que la loi symbolique par laquelle l'immonde peut devenir monde n'a rien à voir avec la loi surmoïque qui en serait plutôt le négatif. Car comme le rappelle Lacan, la loi surmoïque peut se révéler une caricature grimaçante, clownesque, de la loi symbolique :

un énoncé discordant, ignoré dans la loi, un énoncé promu au premier plan par un événement traumatique, qui réduit la loi en une pointe au caractère inadmissible, inintégrable - voilà ce qu'est cette instance aveugle, répétitive, que nous définissons habituellement dans le terme de surmoi (LACAN, 19531954/1975, p. 222)

Vous l'aurez compris, le conformisme est le résultat de la soumission et de l'impossible traitement des injonctions contradictoires du surmoi : « Jouis ! » et « $\mathrm{Ne}$ jouis pas !». À partir de là, le conformiste est coupable. En effet, il n'est pas sans savoir qu'il a cédé sur son désir. Peut-être est-ce pour cela qu'il fait tout pour réduire au silence celui qui se révèle non conforme. Le surmoi quelle qu'en soit la forme sociale - discours fascisants aujourd'hui, nazis hier, inquisiteurs avant-hier vise le même but : faire taire toute voix discordante. Le but étant de faire alors entendre une seule voix afin d'unifier la population. Le procédé, on le sait, est efficace comme l'a démontré son utilisation par Hitler qui promut l'idée

\begin{abstract}
d'un peuple allemand comme un même corps dont il fallait préserver la santé et la pureté (...) ce qui a permis d'unifier une population qui ne l'était pas, une population qui était plutôt marquée par « des différences confessionnelles, la survivance d'un puissant sentiment d'appartenance au Land et à la province, ... la défense acharnée de privilèges sociaux et l'affirmation de subtiles différences dans les habitus respectifs LONGERIC, 2006 ; DE MIOJA, 2016).
\end{abstract}

Ce fantasme de faire de l'Un à partir d'éléments disparates a été décrit par Didier Anzieu sous le nom d'illusion groupale13. Un objet désigné comme mauvais est alors indiqué comme hétérogène au groupe et doit être expulsé pour que tout ce qui est bon, c'est à dire homogène au groupe, soit conservé à l'intérieur : tout ce qui est hétérogène étant rejeté à l'extérieur. Le groupe est ainsi protégé de tout ce qui pourrait

13 Anzieu D. (1981) Le groupe et l'inconscient. Paris, Dunod. 
le contaminer : hétérodoxie de l'hérétique pour l'inquisiteur ou judéité dans le cas du nazisme. Nous retrouvons ici la dynamique décrite par Freud en 1915 dans Pulsions et destin des pulsions à partir de la dynamique entre moi-réel initial et moi-plaisir purifié 14 et qu'il reprendra dix ans plus tard dans son texte sur la dénégation 15 . Pris dans cette dynamique de l'illusion groupale, le groupe devient alors troupeau. Troupeau qui a tôt fait de répondre à la « voix de son maître » en bêlant d'une seule voix 16. Ce qui permet de comprendre que «lorsque que (la voix de) l'objet de vénération disparaît, c'est la panique et l'on pense par exemple à la sidération de beaucoup d'allemands en deuil de leur idéal au lendemain de la mort d'Hitler »17. Dans le même mouvement, Michelet dans son Histoire de la Révolution française 18 relève l'étrange silence qui se fit dans Paris quand le peuple apprit la fuite de Louis XVI à Varennes. Dans un cas comme dans l'autre, la foule reste sans voix face à la disparition de celui qui lui avait permis de se structurer. Le conformiste - fut-il révolutionnaire - est parlé par l'Autre et lorsque celui-ci vient à s'absenter, révélant qu'il n'y a pas d'Autre de l'Autre, il plonge dans un silence où l'immonde, à entendre ici encore comme ce qui n'a pu échoir au symbolique, se fait entendre. Car si

\begin{abstract}
le symbolique détient le pouvoir de symboliser le chaos originaire que le nouveau-né rencontre ; dans cette rencontre où l'immonde va devenir monde humain, le passage de l'un à l'autre ne se fait pas harmonieusement: la métaphore originaire ne symbolise pas tout le réel : un reste demeure en souffrance, et c'est à ce reste en souffrance que le sujet aura affaire dans son destin ( DIDIER-WEILL, 2016, p. 10).
\end{abstract}

C'est dans ce reste, intraité car intraitable, qu'une des faces du surmoi puisera sa dimension forclusive.

\title{
De LA CONFORMité
}

II convient maintenant de montrer en quoi la conformité se distingue du conformisme et nous pouvons précisément le repérer si nous revenons à la proposition

14 Freud S. (1915) «Pulsions et destins des pulsions » dans Métapsychologie. Trad. Fr. CEuvres Complètes, Tome XIII, pais, P.U.F, 2005, p. 182-183.

15 Freud S. (1925) La dénégation. Traduction et commentaires Theves P. et This B. Le Coq-Héron, 1982.

16 La chorégraphie élaborée par les supporters de Jair Messias Bolsonaro illustre cela de façon saisissante. https://www.youtube.com/watch?v=8IWh4WUZHiE

17 De Mijolla S., (2016) opus cité, p. 10. Poizat M., (2001) Vox populi, vox dei. Paris, Métailié.

18 Cité par Didier-Weill A., (1995) Les trois temps de la loi. Paris, Seuil, p. 106.

Psicanálise \& Barroco em revista | v.17, n. 3 | dezembro de 2019 
“II N'y Avait Pas D'autre Choix Possible » : Conformisme, Conformite Et confirmation: Une Approche Psychanalytique Du Devenir-Desistant Ou Du Devenir-Persistant.

de Lacan : «As-tu agi en conformité avec ton désir ? »19. Cette formulation laisse entendre un sens qui ouvre sur la question de l'accord. " As-tu agi en accord avec ton désir ? » pourrions-nous reformuler. Qu'est-ce qu'implique d'agir en accord avec son désir et non d'être en accord avec celui de l'Autre? Lacan nous en donne une illustration tragique à partir de sa lecture de l'Antigone de Sophocle dans la dernière partie de son séminaire VII. Cela est déjà bien connu et a déjà été très largement commenté. Je n'y reviendrai pas. Pour avancer, je vous propose une autre voie qui nous permettra de préciser ce que peut également impliquer la conformité. Je vous propose pour cela de partir du cas du personnage de Melville, Bartleby, et de son célèbre «I would prefer not to » si difficile à traduire. En effet, comment rendre dans une autre langue que celle de Bartleby cette formule que Jean Laplanche propose de qualifier d'affirmation négative?

Bartleby ne dit pas non, il ne dit pas qu'il ne veut pas, il n'hésite pas davantage entre un oui et un non, son « je préférerais » n'implique aucune alternative (je préfère ceci à cela). Dans le I would prefer il y a bien de l'affirmation, mais aussitôt accolée à la négation du not pour s'achever, s'inachever, dans le suspens du not to. Ne pas quoi ? (PONTALIS, 2000, p. 13).

Bartleby est en conformité, pourrions dire, avec son non-désir. II manifeste un absolu «désir de non-désir» pour reprendre la pertinente proposition de Piera Aulagnier2o qui définit ainsi la pulsion de mort. Un mouvement qui viserait le moment où le désir n'avait pas encore eu à s'actualiser. II s'agit là d'un

\footnotetext{
non qui s'énonce d'une voix atone avec une incroyable insistance, une implacable mais toujours calme fermeté, un non qui aurait la douceur d'un oui consentant, un refus de céder à toute demande, qu'elle soit autoritaire, raisonnable, compréhensive, bienveillante ou même des plus affectueuses. Bartleby est intraitable (PONTALIS, op. cit. p. 12) .
}

Cet adjectif d'intraitable est extrêmement intéressant en ce qu'on le rencontre également qualifiant Antigone au vers 472 de la tragédie de Sophocle : « La fille intraitable d'un père intraitable »21. L'agir en conformité avec le désir ouvre ici deux voies qui ont en commun de s'exprimer de façon intraitable. Intraitable : qu'on ne peut pas faire changer d'avis, qui refuse de céder. Les intraitables : ceux qui ne cèdent pas sur leur désir comme ceux qui ne cèdent pas sur leur non-désir. La dimension qui 
apparaît ici est que l'insistance peut parfois se réduire à ce que son étymologie latine peut nous permettre de repérer : -in (à l'intérieur) -sistere (s'arrêter). L'insistance peut alors se transformer en pause, un arrêt proche de la mort où le sujet tenterait de se tenir à distance du désir et de ses turbulences. C'est ici que la conformité sous sa forme insistante peut se révéler ne pas suffire, car elle peut à l'occasion se révéler insistance de la pulsion de mort. II convient alors d'introduire un au-delà de l'insistance qui serait persistance et que je propose de nommer confirmation.

\section{De La Confirmation}

La confirmation est un affermissement et en ce sens, elle est l'expression d'un oui-de-oui. Un oui qui est confirmé par un redoublement22. Elle est persistance au sens où son étymologie nous permet d'y lire une traversée de l'arrêt (-per (à travers) /sistere (l'arrêt)). Cette persistance pourrait être rapprochée du concept freudien, malheureusement si peu fréquenté par les psychanalystes 23, de perlaboration. $\mathrm{Ce}$ rapprochement entre persistance et perlaboration peut s'éclairer à partir de ce que Lacan a pu dire de l'analyse didactique en l'articulant à la perlaboration. Une psychanalyse didactique serait, pour Lacan,

une psychanalyse qui a bouclé cette boucle (du cycle du processus analytique) jusqu'à son terme. II n'y a en effet aucune manière de rendre compte du terme Durcharbeiten, de la nécessité de l'élaboration, si ce n'est à concevoir comment la boucle doit être parcourue plus d'une fois (LACAN, 1964/1996, p. 258).

La confirmation est l'expérience de la persistance qui trouve à s'exprimer dans la dynamique subjective que Lacan a qualifié de s'autoriser et que Alain Didier-Weill modifia en s'auteuriser pour indiquer qu'il s'agit là du moment où le parlant fait le choix de devenir auteur de la parole qu'il a reçue de l'Autre. Le oui-de-oui est lié à la persistance du désir malgré le censeur surmoïque, le conformisme de groupe et / ou la peur du tyran quand le oui du conformiste se révèle acquiescement à la soumission et désistance.

22 C'est bien là le sens du sacrement de la confirmation pour les catholiques.

${ }_{23}$ Cela est d'autant plus étonnant que Freud fait de la perlaboration ce qui distingue la cure analytique de toutes les autres pratiques psychothérapeutiques. Freud S., (1914) «Remémoration, répétition et perlaboration », CEuvres complètes, Tome 12, Paris, P.U.F. 2005, p. 185-196.

Psicanálise \& Barroco em revista | v.17, n. 3 | dezembro de 2019 
“II N'y Avait Pas D'autre Choix Possible » : Conformisme, Conformite Et confirmation: Une Approche Psychanalytique Du Devenir-Desistant Ou Du Devenir-Persistant.

Le devenir-persistant, dont on peut espérer qu'il concerne l'analyste, implique cette assomption du oui-de-oui de la persistance mais rien ne garantit le passage de l'insistance à la persévérance et donc sa confirmation. Comment le sujet peut-il assumer et valider cette position malgré sa constitution sur fond d'immonde? Comment peut-il dire « oui » à un monde qui reste topologiquement en rapport avec l'immonde ? Ce « oui » qui peut se produire dans une psychanalyse est, comme l'a montré Alain Didier-Weill, un «oui » dont la complexité tient à l'articulation antinomique qu'il doit produire : le sujet doit dire « oui » à ce qu'il y a de plus réel en lui, de non advenu au symbolique («Je ne suis que ça », ce qui relève de l'immonde) et dire « oui » à ce qui, en lui, contredit le réel (« Je ne suis pas que ça ») en advenant au symbolique. Ce «oui-de-oui », par lequel le sujet assume d'être, dans un même mouvement, et «ça » et « pas que ça », est l'acte par lequel il assumera sa division d'être parlant ce qui permettra que le « s'autoriser de » ne soit pas « un s'autoriser par ». « Le psychanalyste a horreur de son acte» nous avertit Lacan.

Qu'est-ce qu'il y a de si horrible dans cet acte qui consiste à s'autoriser au point que certains analystes voudraient l'éviter en le convertissant en acte d'obéissance à une école, un maître, voire un Messie? Rien d'autre que la castration symbolique, ce point d'où le réel troue le symbolique et d'où ek-siste le désir de l'analyste, comme d'ailleurs celui de l'analysant, si bien que l'acte de s'autoriser s'articule ainsi : « comment savoir si je suis fidèle à moi-même, à ce qui est advenu dans mon analyse, quand je dis être devenu analyste ? »24. Celui qui recule devant l'acte au sens analytique du terme préfère l'obéissance à une règle qui lui dit quoi faire au risque de se ritualiser plutôt que la loi qui le conduit à devoir improviser25 dans son sens le plus noble. En effet comme le rappelle Lacan, dans la Note italienne, « s'autoriser n'est pas auto-ri(tuali)ser» 26 auquel cas l'autorisation ne serait qu'un autre nom du conformisme, ce que Freud avait déjà pu repérer comme nous l'avons rappelé précédemment.

Alors qu'est-ce que serait la confirmation et le devenir-persistant dans ce contexte ? Pour répondre rapidement à cette question et conclure, je vous propose, en reprenant le célèbre aphorisme freudien, la formulation suivante : « Là où c'était, je dois me confirmer en m'auteurisant». L'auteurisation serait alors à comprendre 
comme le devenir-persistant, autre nom de la perlaboration, au lieu même de l'inconscient où la confirmation se révèle confirmation de la parole reçue et accueillie. Après tout, le sujet ne saurait être qu'un « homme de parole ». Au sens où le sujet tient à elle, par elle et en assume les conséquences qui font que face à la tentation toujours présente du choix de l'immonde, le choix de la fidélité à la parole, dans une relance incessante, nous rappelle qu'un monde habitable et partageable est possible. Car comme nous le rappelle Marc-Alain Ouaknin de façon provocatrice et quasi hérétique, «Le Messie est fait pour ne pas venir » 27, nous offrant dans sa non-venue le vide de l'attente créatrice où le sujet est condamné à s'auteuriser ou à disparaître. 
“II N'y Avait Pas D'autre Choix Possible »: Conformisme, Conformite Et confirmation:

Une Approche Psychanalytique Du Devenir-Desistant Ou Du Devenir-Persistant.

\section{REFERÊNCIAS}

ANZIEU D., Le groupe et l'inconscient. Paris, Dunod, 1981.

ARENDT H., (1963) Eichmann à Jérusalem. Paris, Gallimard/Folio. 2002.

AULAGNIER P., La violence de l'interprétation. Paris, P.U.F, 1975.

BAYARD P. Aurais-je été résistant au bourreau? Paris, Editions de minuit, 2003.

BROWNING C., (1992) Des hommes ordinaires. Paris, Tallandier /Texto, 2007.

DE MIJOLLA S. «Le Conformisme en politique, dans l'éducation et en psychanalyse ». Topique, Conformisme et conservatisme, 2016, p. 7-20.

DIDIER-WEILL A., "Bénir, Maudire ou mi-dire Lacan ?”, Esquisses psychanalytiques. n 15 , 1991, p. 77-86.

DIDIER-WEILL A., Les trois temps de la loi. Paris, Seuil, 1995.

DIDIER-WEILL A., Qu'est-ce que le surmoi. Toulouse, Eres, 2016.

FREUD S., (1909-1939) Correspondance avec le pasteur Pfister, lettre du 25/11/1928. Paris, Gallimard, 1966.

FREUD S., (1914) « Remémoration, répétition et perlaboration », Euvres complètes, Tome 12, Paris, P.U.F. 2005, p. 185-196.

FREUD S., (1915) «Pulsions et destins des pulsions » dans Métapsychologie. Trad. Fr. Euvres Complètes, Tome XIII, Paris, P.U.F, 2005, p. 163-187.

FREUD S., (1925) La dénégation. Traduction et commentaires Theves P. et This B. Toulouse, Le Coq-Héron, 1982.

JONES E., (1955) La vie et l'œuvre de Sigmund Freud, tome 2 : Les années de maturité. Paris, PUF, 2006.

LACAN J., (1953-1954) Le Séminaire, Livre 1, Les écrits techniques de Freud. Paris, Seuil, 1975.

LACAN J., (1955) «La chose freudienne ou Sens du retour à Freud en psychanalyse », Écrits. Paris, Seuil, 1966.

LACAN J., (1959-1960) O seminário, livro 7, A ética da psicanálise. Rio de Janeiro, Jorge Zahar Editor, 1997.

LACAN J., (1964) O Seminário, Livro 11, Os quatro conceitos fundamentais da psicanálise. Rio de Janeiro, Jorge Zahar Editor, 1996. 
LACAN J., Dissolution, Journal le Monde, 26 janvier 1980.

LACAN J., (1982) « Note italienne », Autres écrits. Paris, Seuil, 2001.

LIPPI S., Vinot F., Cliniques Méditerranéennes $\mathrm{n}^{\circ} 93$ : Improviser en psychanalys(t)e. Toulouse, Eres. 1996.

LONGERICH P. (2006) Nous ne savions pas. Paris, Éd. Héloïse d’Ormesson, 2006.

MILGRAM S., Soumission à l'autorité. Paris, Fayard/Pluriel, 2017.

OUAKNIN M.-A. Bibliothérapie. Lire c'est guérir. Paris, Seuil, 1994.

PONTALIS J-B, «L'affirmation négative », Libres cahiers pour la psychanalyse, vol. 2, no. 2, 2002.

SOPHOCLE, Antigone. Trad. Fr. Paul Mazon. Paris, Gallimard, 1973.

VINOT F., Vives J.-M., (2016) «Conformisme de vie, conformisme de mort», Topiques, Conformisme et conservatisme, $\mathrm{n}^{\circ} 136$. p. 91-101. 


\title{
“It Didn't Have Other Possible Choice”: Conformism, CONFORMity AND CONFIRMATION - A PSYCHOANALYTICAL Approach Of The Becoming-Desistent Or Of The Becoming- Persistent
}

\begin{abstract}
Starting from a precise differentiation of the meaning of the terms of conformism, conformity and confirmation, the author proposes to describe the psychic mechanisms present in the becoming-desistent (which implies desisting, giving up one's desire) and in the becoming-persistent (which is related to the perlaboration of the desire). This path from the conformism to the confirmation allow us to precise the Lacanien aphorism correlated to the ethic of the psychoanalysis: "Have you acted in conformity with your desire?".
\end{abstract}

KEY WORDS: conformism, conformity, confirmation, desire, ethic, voice. 


\section{“Não Havia Outra Escolha Possível": Conformismo, CONFORMIDAdE E CONFIRMAÇÃo - UMA ABORDAGEM Psicanalítica Do DeVIR-Desistente Ou Do DeVIR-PERSISTENTE}

\section{Resumo}

A partir de uma diferenciação precisa do sentido dos termos de conformismo, conformidade e confirmação, o autor se propõe a descrever os mecanismos psíquicos em jogo no devir-desistente (o que implica em ceder de seu desejo) e no devirpersistente (que teria a ver com a perlaboração do desejo). Este percurso do conformismo à confirmação permite precisar o aforismo lacaniano em relação à ética da psicanálise: "Agistes em conformidade com teu desejo?".

Palavras-Chave: Conformismo, Conformidade, Confirmação, Desejo, Ética, Supereu, Voz. 
«II N'y Avait Pas D'autre Choix Possible » : Conformisme, Conformite Et confirmation: Une Approche Psychanalytique Du Devenir-Desistant Ou Du Devenir-Persistant.

RECEBIDO EM 26-09-2019

APROVADO EM 20-10-2019

(C) 2019 Psicanálise \& Barroco em revista

http://www.seer.unirio.br/index.php/psicanalise-barroco/index

revista@psicanaliseebarroco.pro.br

Programa de Pós-Graduação em Memória Social — UNIRIO

Memória, Subjetividade e Criação

www.memoriasocial.pro.br/proposta-area.php 